\title{
Energy-efficient radio-over-fiber system for next-generation cloud radio access networks
}

\author{
Bo Wang ${ }^{1}$, Limei Peng $2^{*}$ (D) and Pin-Han Ho ${ }^{1}$
}

\begin{abstract}
The paper proposes a novel adaptive radio-over-fiber (RoF) system for next-generation cloud radio access network (C-RAN), aiming to optimize the operation cost in terms of power consumption while maintaining required data rate. By jointly considering the nonlinear distortion from Mach-Zehnder modulator (MZM) and high power amplifier (HPA) due to high peak-to-average-power ratio (PAPR) in the electronic domain, we first provide a $2 \times 2$ multiple-input mulitple-output orthogonal frequency division multiplexing (MIMO-OFDM) baseband model on electrical SNR (ESNR) for a single RoF transmission line. To take the modulation levels into consideration, we provide the optical signal to noise ratio (OSNR) analysis that jointly considers the electrical SNR (ESNR) model and the non-linear effect of the optical transmission. This optical SNR (OSNR) analysis result is further used in the subsequent power consumption model for both the downlink and uplink of the considered RoF transmission system. Case studies via simulation and numerical experiments are conducted to verify that the proposed RoF system not only can reach the lowest power and spectrum consumptions at same time, but also consumes considerably less power than current RoF system.
\end{abstract}

Keywords: Radio-over-Fiber (RoF), Cloud radio access network (C-RAN), Energy efficiency, Peak-to-average power ratio (PAPR), Nonlinear distortion

\section{Introduction}

Today's wireless traffic is dominated by IP-based multimedia services and applications, which have caused significant burdens on the radio access networks (RANs). Enlarging the system capacity is a simple remedy that could nonetheless cause poor equipment utilization and low energy efficiency at the base stations (BSs) due to high traffic fluctuation and network dynamics. The stringent demand on quality of service (QoS) further leaves the legacy RANs an awkward situation in dealing with the application scenarios envisioned in the near future.

Cloud RAN (C-RAN), defined with LTE-Advance and those under the banner of $5 \mathrm{G}$, aims to resolve the bandwidth thirsty of the current RANs while achieving high network responsiveness and QoS provisioning. With channel bandwidths up to $100 \mathrm{MHz}$ and the downlink peak rates of $1 \mathrm{~Gb} / \mathrm{s}$, four radio channels per direction

*Correspondence: auroraplm@knu.ac.kr

${ }^{2}$ School of Computer Science and Engineering, Kyungpook National

University, 41566 Daegu, Republic of Korea

Full list of author information is available at the end of the article are provisioned at each BS, which consumes a minimal serial bit rate of $24 \mathrm{~Gb} / \mathrm{s}$ in the backhaul and up to 100 $\mathrm{GB} / \mathrm{s}$ is foreseen in the near future [1]. The deployment of densely distributed and centralized-controlled low-power small cells can better handle the high traffic fluctuation via fast reconfiguration [2]. This is realized by equipping the central unit $(\mathrm{CU})$ with multi-site and multi-standard baseband units, such that multiple wireless standards for geographically dispersed BSs with different levels of sectors and coverage can be supported. To this end, all the advanced technologies in the conventional RANs, such as enhanced MIMO, co-operative multi-point (CoMP), carrier aggregation, and strategies toward network heterogeneity, are modified from a distributed version to fit into a highly centralized control paradigm.

Nonetheless, the common public radio interface (CPRI) defined under LTE advanced can only support up to 6.144 $\mathrm{Gb} / \mathrm{s}$ [3] and is merely adequate for a few $20 \mathrm{MHz}$ channels, or a single $100 \mathrm{MHz}$ radio channel [4], thus could easily render a bandwidth bottleneck. Featured by link transparency and lower bandwidth requirement per BS
Springer Open

(c) The Author(s). 2019 Open Access This article is distributed under the terms of the Creative Commons Attribution 4.0 International License (http://creativecommons.org/licenses/by/4.0/), which permits unrestricted use, distribution, and reproduction in any medium, provided you give appropriate credit to the original author(s) and the source, provide a link to the Creative Commons license, and indicate if changes were made. 
comparing to current CPRI-based digital transmission approaches, radio over fiber (RoF) systems are considered as a good counterpart for centralized wireless infrastructure of C-RAN in the next-generation mobile communications.

This paper investigates an energy-efficient RoF system as the backbone transmission technique for the C-RAN [5]. Different from all the previously reported research, the proposed RoF system employs a $2 \times 2$ MIMO module and coherent optical orthogonal frequency division multiplexing (CO-OFDM) transmission technology. Thanks to the partially overlapped subcarriers and high tolerance to chromatic dispersion (CD) and polarization mode dispersion (PMD), the CO-OFDM transmission system demonstrates superb spectrum efficiency and bandwidth slicing flexibility. By provisioning radio access via $\mathrm{CO}$ OFDM-based optical channels, the resultant RoF system is shown with great potential to achieve improved service provisioning granularity without losing data capacity compared with legacy WDM-based RoF systems.

The rest of the paper is organized as follows. Section 2 provides a comprehensive review on the enabling technologies for the proposed RoF system. Section 3 presents the proposed RoF system for next-generation C-RAN and provides a multi-input-multi-output OFDM (MIMOOFDM) baseband model for simulating the end-to-end RoF system, followed by OSNR calculation and power consumption analysis in Section 4. Section 5 shows the case study results that compares the proposed MIMOOFDM transmission system. Section 6 concludes the paper.

\section{An overview}

The section provides a high-level overview on the proposed research regarding C-RAN and RoF.

\subsection{Cloud radio access network (C-RAN)}

Under the current distributed antenna systems (DAS) architecture, each BS serves as a layer-2 switch by providing baseband processing functions and is further connected to the remote radio unit (RRUs) via a high-speed link, also referred to as front-haul. Such a distributed network architecture has been considered short of flexibility and dynamic configurability for fluctuating traffic demand and multiple wireless standards, coverage, and frequency spectrum [6]. Further, since a cooling system has to be in each BS due to the presence of the processing unit, nontrivial power consumption is incurred in presence of a large number of cells.

C-RAN mitigates the abovementioned problems by centralizing the baseband processing functions in the centralized unit, in which a processing unit pool is created in order to accommodate the baseband processing units of all the BSs under its cell site. With this, the capacity of baseband units (BBUs) pooled at the central unit can be dynamically and elastically allocated with sufficient flexibility. In the meantime, advanced co-processing and synchronization techniques can be easily deployed due to the centralized and pooled BBUs, such as heterogeneous networks and co-operative multi-point (CoMP). On the other hand, the RRUs of each BBU are distributed across a wide area and are connected with the BBU via high-speed links, which are also referred to as front-hauls. According to the current industry practice, common public radio interface (CPRI) protocol, defined under 3GPP, is used on optical fibers to support these front-haul links.

The CRPI front-hauls and the C-RAN backbone are generally supported by WDM technology for exploring larger capacity and guaranteed QoS. Due to the highly dynamic environment, nonetheless, the WDM-based CRAN could waste a significant amount of energy and be subject to low channel utilization on optical transmissions that have to over-provision to meet the stringent QoS and bandwidth requirements. Another problem due to the use of digital front-haul link is caused by analogdigital conversion that naturally increases the end-to-end latency and hardware complexity. Thus, making the optical transmissions adaptive and software programmable according to the traffic variation is critical to the design of the next-generation C-RANs. It has been widely reported that we can dynamically manipulate a number of configurations/parameters of an optical OFDM transmission line to achieve better energy and spectral efficiency [7], and these parameters include the modulation format, the code rate of the forward error correction (FEC), the symbol rate (per subcarrier), the number of polarizations per wavelength, and the number of OFDM subcarriers. Such adaptive transceivers are expected to efficiently allocate spectral bandwidth in the presence of highly dynamic traffic demands.

\subsection{Radio-over-fiber (RoF)}

Instead of conventional CPRI-based digital link, the study considers the RoF technique for the high-speed fronthaul link in-between the central unit BBU pool and each remote radio unit (RRU). An RoF system is characterized by simple and lower-power RF transmitters as well as capability for dynamic resource allocation and mobility management [8], which demonstrates numerous merits against its counterparts. In spite of smaller radio ranges due to limited amplification function at the RF transmitter, it offers very low implementation cost and is considered more suitable for supporting multiple wide-band radio channels than the CPRI [4].

Coherent optical OFDM-based MIMO-RoF is an advanced implementation of the RoF technique [9], thanks to the robustness of MIMO channel and the nature of multiple access in employing OFDM (or referred to 
as OFDMA). It is clear that transporting multiple subcarriers at the same wavelength (i.e., SCM) and possibly multiple wavelengths multiplexed (i.e., WDM) through the RoF links is more challenging than the CPRI transmission and thus takes more careful designs and control/management.

Similar to digital CPRI, a RoF link is typically subject to optical component nonlinearities and fiber chromatic dispersion, as well as difficulties in implementing remote control with a large number of RRUs. Although these limitations can be overcome by providing dedicated wavelengths to each RRU, it turns out to be a rather costly solution subject to a scalability problem when the number of RRUs is increasing. To provide fine granularity of bandwidth without losing scalability, a RoF transmitter could correspond to multiple RRUs by implementing a point-tomultipoint optical network architecture, such as passive optical networks (PONs). As shown in Fig. 1, long-reach bidirectional RoF transmission system was demonstrated in [10], where the $65 \mathrm{~km}$ feeder extension of the single mode fiber (SMF) type is added to the PON with passive splitters for multi-wavelength overlay.

\section{Proposed RoF transmission system for next-generation C-RAN}

It is clear that the state-of-the-art RoF schemes, such as in [10], aim to support a rather small distribution range (e.g., $<85 \mathrm{~km}$ ), conservative modulation schemes (e.g., 64-QAM or lower), and narrow channel bandwidth for downstream $(20 \mathrm{MHz})$, which fail to serve in $5 \mathrm{G}$ mobile systems targeting wider channel bandwidth (100 MHz) and higher levels of modulation scheme (256-QAM). To meet the requirements of future C-RAN, the paper investigates an advanced RoF transmission system. As shown in Fig. 2, the proposed $2 \times 2$ MIMO-RoF system is featured with polarization division multiplexing (PDM) technology for increasing the number of provisioned BSs, as well as coherent optical (CO) detection [11] at the receiver for achieving high data rate and long distance transmission. One most important feature is that the transmit power, modulation scheme, and the number of subcarriers for each optical layer transmission can be adaptively determined and software programmed. With this, an intelligent configuration strategy should be in place in order to make transmission efficient and effective.

Note that the employment of coherent detection using local oscillator (LO) laser in the receiver, although signifiantly improved the transmission quality, will inevitably raise the system cost. Thus, the proposed system is suitable for provisioning large-scale antenna arrays that are over several tens of kilometers from the baseband unit (BBU) pool.

The rest of the section presents the mathematical expressions of some important devices along the data path.

\subsection{Baseband models for RoF transmission system}

As shown in Fig. 3, a $2 \times 2$ MIMO-OFDM baseband model for simulating the required ESNR of end-to-end RoF transmission system is developed by jointly considering nonlinear distortion from both Mach-Zehnder modulator (MZM) and high power amplifier (HPA) due to high PAPR.

\subsection{PAPR in OFDM}

Consider an OFDM transmission with $L$ subcarriers at the frequencies $\left\{f_{l}, l=1, \cdots, L\right\}$. Assigned to the subcarriers at $\left\{f_{l}, 1 \leq l \leq L\right\}$ are, respectively, the $M$-ary data symbols $\left\{\bar{x}_{l}, l=1, \cdots, L\right\}$ or 0 , which are independent and identically distributed random variables with zero mean and variance $P$.

Let $T$ be the modulation interval and $L T$ be the duration of an OFDM symbol (excluding the guard interval). The OFDM-signal's complex envelope can be expressed as:

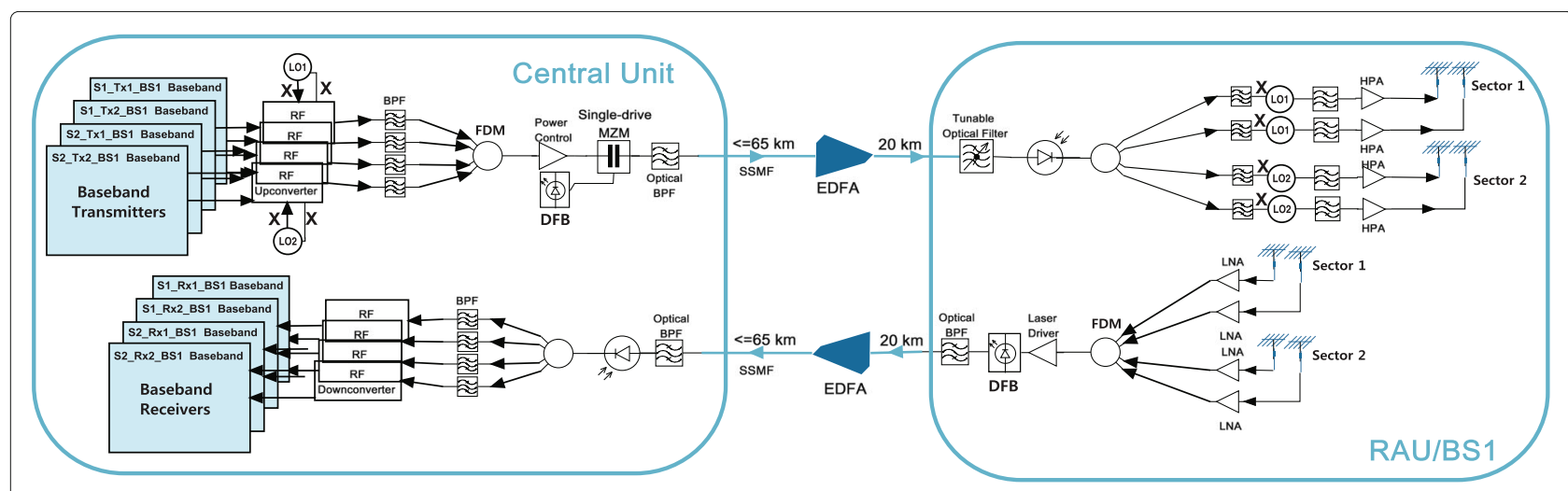

Fig. 1 Current RoF transmission system 


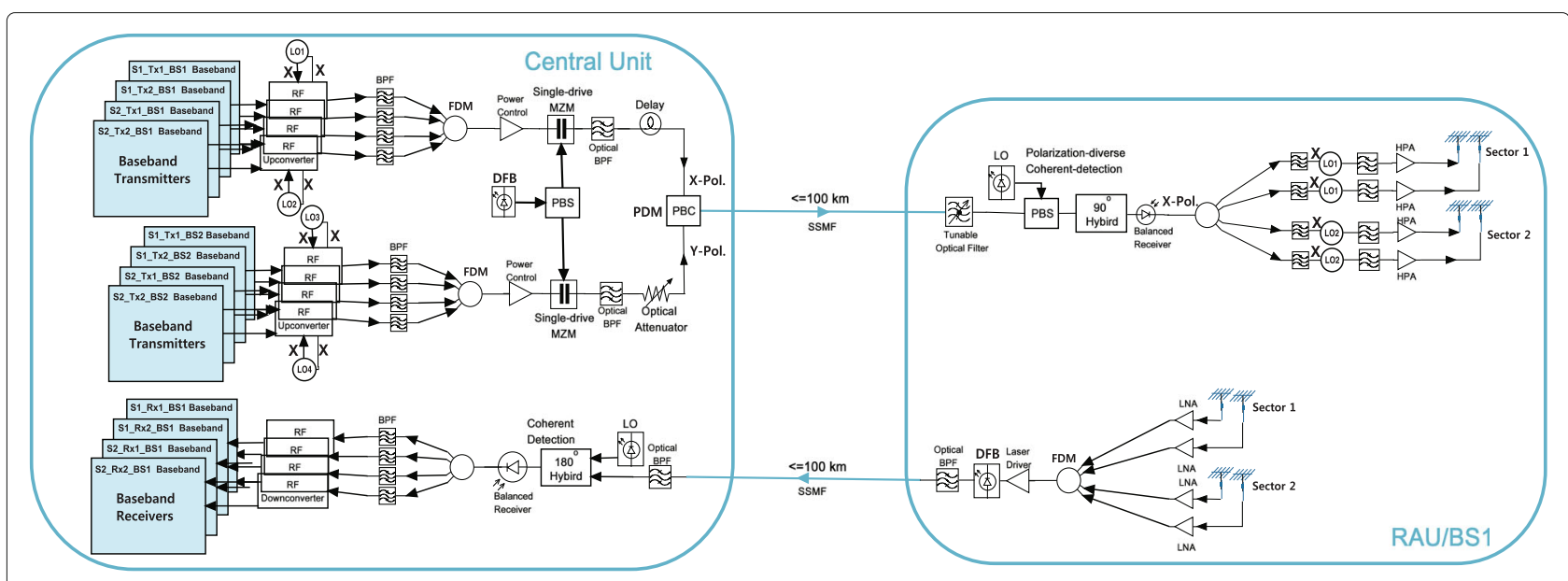

Fig. 2 Proposed RoF transmission system for next-generation C-RAN

$$
x(t)=\frac{1}{\sqrt{L}} \sum_{l=1}^{L} \bar{x}_{l} e^{j 2 \pi f_{l} t}, \quad 0 \leq t \leq L T .
$$

assuming the above is an idealized rectangular timedomain window, and the cyclic-prefix extension of $x(t)$ would not alter the PAPR.

The PAPR of the continuous-time signal $x(t)$ is defined as:

$$
\zeta(x(t)) \stackrel{\text { def }}{=} \frac{\max _{0 \leq t<L T}\left\{|x(t)|^{2}\right\}}{E\left[|x(t)|^{2}\right]}
$$

where $E[\cdot]$ denotes the expectation value.

\subsection{Electrical power amplifier model}

A memoryless nonlinear HPA with a "soft limiter" inputoutput relationship is employed. For a complex-value input $y$, the output equals $\Lambda(|y|) e^{j<y}$ [12], where:

$$
\Lambda(a)=\left\{\begin{array}{l}
a, \text { if } a \leq A, \\
A, \text { if } a>A .
\end{array}\right.
$$

The "clipping ratio" is defined as $\gamma=\frac{A}{\sqrt{P_{\text {in }}}}[12]$, where $P_{\text {in }}$ denotes the average power of the input signal, $\gamma=3 \mathrm{~dB}$.

\subsection{Optical MZM model}

As a waveguide-based external modulator, MZM is a device widely employed in optical OFDM systems with chirp-free signals for achieving high data rate transmissions. As shown in Fig. 2, the optical OFDM signal is modulated based on the electrical OFDM signal by using the MZM. The modulation process is nonetheless subject to nonlinear and peak-limited transfer characteristics.

When using differential input data in a push-pull configuration, the transfer function of a single-drive MZM is given by [13]:

$$
\frac{E_{\text {out }}(t)}{E_{\text {in }}(t)}=\cos \left(\frac{\pi V(t)}{2 V_{\pi}}\right)
$$

where $E_{\text {in }}(t)$ and $E_{\text {out }}(t)$ are the input and output in optical field, respectively; $V(t)$ is the electrical OFDM signal; and $V_{\pi}$ is the required voltage difference applied to a single



Fig. $32 \times 2$ MIMO-OFDM Baseband Model for RoF Transmission System 
electrode in order to generate a phase shift between two waveguides.

Expanding the MZM nonlinear transfer function into a Taylor series as:

$$
\frac{E_{\text {out }}(t)}{E_{\text {in }}(t)}=\cos \left(\frac{\pi V(t)}{2 V_{\pi}}\right) \approx 1-\frac{1}{2 !}\left(\frac{\pi V(t)}{2 V_{\pi}}\right)^{2}+\ldots
$$

The baseband equivalent polynomial model for the output electrical field of the MZM is given as:

$$
\begin{aligned}
y_{k} & =\sum_{q=1}^{Q} \alpha_{q} \cdot\left(x_{k}\right)^{Q}, q-o d d \\
& \approx \alpha_{1} \cdot x_{k}+\alpha_{3} \cdot\left(x_{k}\right)^{3}
\end{aligned}
$$

where $x_{k}$ and $y_{k}$ are the discrete vectors of the applied voltage and the output voltage at the MZM, respectively; $q$ is the order of nonlinearity; and $\alpha_{q}$ is the odd coefficient of the MZM nonlinear transfer function with an operating region $3 V_{\pi} \pm V_{\pi}$ (i.e., at the null intensity bias point), whose output signal is approximated as a third order polynomial [14].

\subsection{Optical SNR calculation}

By assuming an ideal detection of optical OFDM-MIMO system and the line-widths of the transmit/receive lasers to be zero, the study takes the relation between the SNR in the optical domain (OSNR) and the electrical SNR (ESNR) at ideal coherent receiver as follows [15]:

$$
\operatorname{OSNR}(M)=\operatorname{ESNR}(M) \frac{D}{2 B_{\text {ref }} \cdot m_{\text {sys }}}
$$

where $B_{\text {ref }}$ is the reference bandwidth used for the OSNR measurement $(\approx 12.5 \mathrm{GHz}$ for $0.1-\mathrm{nm}$ bandwidth around $1550 \mathrm{~nm}$ ); $M$ is the constellation size of $M$-ary quadrature amplitude modulation (QAM); $m_{\text {sys }}$ is the system margin $\approx 12 \mathrm{~dB}$ [16]; and $D \mathrm{~Gb} / \mathrm{s}$ is the total system symbol transmission rate. And, this relationship is independent of whether using polarization multiplexing or not [15].

As a RoF downstream transmission line shown in Fig. 1, the available OSNR of a $0.1-\mathrm{nm}$ band at around $1550 \mathrm{~nm}$ at the optical receiver can be given by:

$$
\begin{aligned}
\text { OSNR }= & P_{\text {out }}-L_{M Z M}-L_{\text {aligned }}-L_{\text {Pol }}-L_{O B P F} \\
& -L_{T O F}-\alpha_{\text {span }}-F_{E D F A}+G_{E D F A}-G_{R x} \\
& -L_{\text {other }}
\end{aligned}
$$

where $P_{\text {out }}$ is output power of distributed feedback laser (DFB) laser source up to $16 \mathrm{dBm}$. $L_{M Z M}$ is optical excess loss of MZM with a typical value $7.75 \mathrm{~dB}$ for 4 channels multiplexing; $L_{\text {aligned }}$ is passive splitter loss (1:16) with a typical value $14 \mathrm{~dB}$ [10]; $L_{O B P F}$ is optical band-pass filter loss with a typical value $3 \mathrm{~dB} ; L_{T O F}$ is tunable optical filter (or add/drop multiplexer) loss with a typical value $5 \mathrm{~dB}$ [17]; and $\alpha_{\text {span }}=17 \mathrm{~dB}$ is the loss of $85-\mathrm{km}$ SSMF span $(0.2 \mathrm{~dB} / \mathrm{km})$ [17]. For a typical EDFA, $F_{E D F A}$ is the
EDFA noise figure due to the ASE noise with a typical value of $6 \mathrm{~dB} ; G_{E D F A}$ is the EDFA gain up to $20 \mathrm{~dB}$. $G_{R x}$ is receiver sensitivity no better than $-30 \mathrm{dBm}$ for the avalanche photodiode (APD) [17]. Some other elements along the datapath of Fig. 1 may affect the end-to-end dB values, including circulator loss and array waveguide grating (AWG) loss, are jointly represented as $L_{\text {other }}$, which takes a values of $5.76 \mathrm{~dB}$. Note that although we have considered all possible elements to the best of our survey, there could be more elements along the data path that affect the $\mathrm{dB}$ values. The proposed power consumption model, nonetheless, is generic to a RoF system and can be easily expanded according to any addition upon the system.

For the PDM-based RoF downstream transmission line in Fig. 2, the available OSNR of a $0.1 \mathrm{~nm}$ band at around $1550 \mathrm{~nm}$ at the coherent receiver can be given by:

$$
\begin{aligned}
\text { OSNR }= & P_{\text {out }}+P_{L O}-L_{P D M}-2 L_{C i r}-L_{\text {aligned }} \\
& -L_{O B P F}-L_{T O F}-\alpha_{\text {span }}-G_{C o-R x}
\end{aligned}
$$

where $P_{L O}$ is output power of local oscillator (LO, DFB laser) at coherent receiver with value up to $16 \mathrm{dBm}$. $G_{C o-R x}$ can achieve a sensitivity of $-45.9 \mathrm{dBm}$ in LR-PON over $100-\mathrm{km}$ SSMF by using coherent receiver without amplification [11]. $L_{P D M}$ is the PDM loss with a typical value $24.9 \mathrm{~dB}$, including laser power splitter, MZM loss, AWG loss, polarization beam splitter, variable attenuator, and polarization beam combiner [16].

\section{Power consumption analysis}

Based on the ESNR requirement simulated by MIMOOFDM baseband model, we use an ideal ESNR-to-OSNR transfer function to derive the corresponding OSNR for supporting the ESNR requirement. The relation between the required ESNR and OSNR is provided based on some assumptions. The OSNR is further derived to calculate the required output power of laser source. Then, we propose the power consumption model for the optical part of RoF transmission system.

\subsection{Power consumption analysis}

The general power consumption of optical transmitter $P_{T x}$ (in Watt) is given by:

$$
\begin{aligned}
P_{T x}= & \gamma_{D C}^{-1}\left(P_{\text {laser }}+P_{\text {map }}+2 P_{P / S}+n_{D A C} \cdot P_{D A C}\right. \\
& \left.+n_{M Z M} \cdot P_{M Z M}+P_{I F F T-C P-T S}\right)
\end{aligned}
$$

where $\gamma_{D C}$ is the power conversion efficiency of converting the $+12 \mathrm{~V}$ DC power supply for transceiver modules with a value $93 \% ; P_{\text {map }} \approx(0.019 \cdot D) / \log _{2}(M)$ is the power consumption for signal mapping by different modulation level $M ; P_{P / S}=0.02 \cdot D$ is the power consumption for parallel-to-serial (or serial-to-parallel) conversion; $P_{D A C} \approx(0.008 \cdot D) / \log _{2}(M)$ is the power consumption 
for a single digital-to-analog converter (DAC); $P_{M Z M} \approx$ $0.017 \cdot D$ is the power consumption for a single-drive MZM; and $P_{I F F T-C P-T S} \approx(0.16 \cdot D) / \log _{2}(M)$ is the power consumption for the IFFT, CP, and TS modules for 512 OFDM subcarriers [16]. $n_{D A C}$ and $n_{M Z M}$ are the number of required DACs and MZMs by different RoF systems, respectively.

The general power consumption of optical receiver $P_{R x}$ (in Watt) is given by:

$$
\begin{aligned}
P_{R x}= & \gamma_{D C}^{-1}\left(P_{L O}+P_{T I A}+n_{P D} \cdot P_{P D}\right. \\
& \left.+n_{A D C} \cdot P_{A D C}+P_{R x-D S P}\right)
\end{aligned}
$$

where $P_{L O}$ is the power consumption for the local oscillator $(\mathrm{LO})$ at coherent receiver equals to the $P_{\text {laser }}$; $P_{T I A}=$ $(0.0188 \cdot D) / \log _{2}(M)$ is the power consumption of transimpedance amplifier with automatic gain control for current-to-voltage conversion; $P_{P D} \approx 0.0028 \cdot P_{C W} \cdot D$ is the power consumption for a single photodiode (PD); $P_{A D C} \approx(0.0175 \cdot D) / \log _{2}(M)$ is the power consumption for a single analog-to-digital converter (ADC); and $P_{R x-D S P} \approx(0.36 \cdot D) / \log _{2}(M)$ is the power consumption for the DSP module of signal post-processing at receiver for $100 \mathrm{~km}$ distance and 512 OFDM subcarriers [16]. $n_{A D C}$ and $n_{P D}$ are the number of required ADCs and photodiodes by different RoF systems, respectively.

The power consumption of the laser $P_{\text {laser }}$ (in Watt) for downlink in $\mathrm{CU}$ is calculated by:

$$
\begin{aligned}
P_{\text {laser }} & =E_{\text {Laser }} \cdot 10^{-3} \cdot D_{\text {down }}+P_{C W} \\
& =E_{\text {Laser }} \cdot 10^{-3} \cdot D_{\text {down }}+10^{\left(P_{\text {out }}-30\right) / 10}
\end{aligned}
$$

where $P_{C W}$ (in Watt) derived from $P_{\text {out }}$ (in $\mathrm{dBm}$ ) is the laser continuous wave $(\mathrm{CW})$ output power; $D_{\text {down }}$ is downstream data rate; and $E_{\text {Laser }}$ is the DFB laser energy consumption with a typical value as $1.5 \mathrm{pJ}$ per bit for supporting up to $40 \mathrm{~Gb} / \mathrm{s}$ [18]. For a ESNR value, $P_{\text {out }}$ is calculated based on (6), (7), or (8) by taking $M$ as variables.

The power consumption of the laser equipped with driver (instead of MZM) $P_{\text {laserDr }}$ (in Watt) for uplink is calculated by:

$$
\begin{aligned}
P_{\text {laserDr }} & =E_{\text {LaserDr }} \cdot 10^{-3} \cdot D_{\text {up }}+P_{C W} \\
& =E_{\text {LaserDr }} \cdot 10^{-3} \cdot D_{\text {up }}+10^{\left(P_{\text {out }}-30\right) / 10}
\end{aligned}
$$

where $D_{u p}$ is the upstream data rate and $E_{L a s e r D r}$ is the energy consumption by the DFB laser with a typical value of $37 \mathrm{pJ}$ per bit for supporting up to $40 \mathrm{~Gb} / \mathrm{s}$ [18].

The power consumption of a link EDFA $P_{E D F A}$ per wavelength is given by [16]:

$$
\begin{aligned}
P_{E D F A} & =\gamma_{E D F A}^{-1}\left(\operatorname{ESNR}(M) \cdot 1.58 \cdot\left(e^{\alpha_{\text {span }}}-1\right)\right. \\
& \left.\cdot\left(1-e^{-\alpha_{\text {span }}}\right) h v\right) \cdot 10^{9} \cdot D \\
& +\left(P_{\text {EDFAoh }} \cdot D\right) / 100
\end{aligned}
$$

where $\gamma_{E D F A}$ is the conversion efficiency of EDFA power with a value $2 \% ; P_{E D F A o h}$ is the power consumption of EDFA overhead with a value $0.69 \mathrm{~W} ; h$ is a constant with

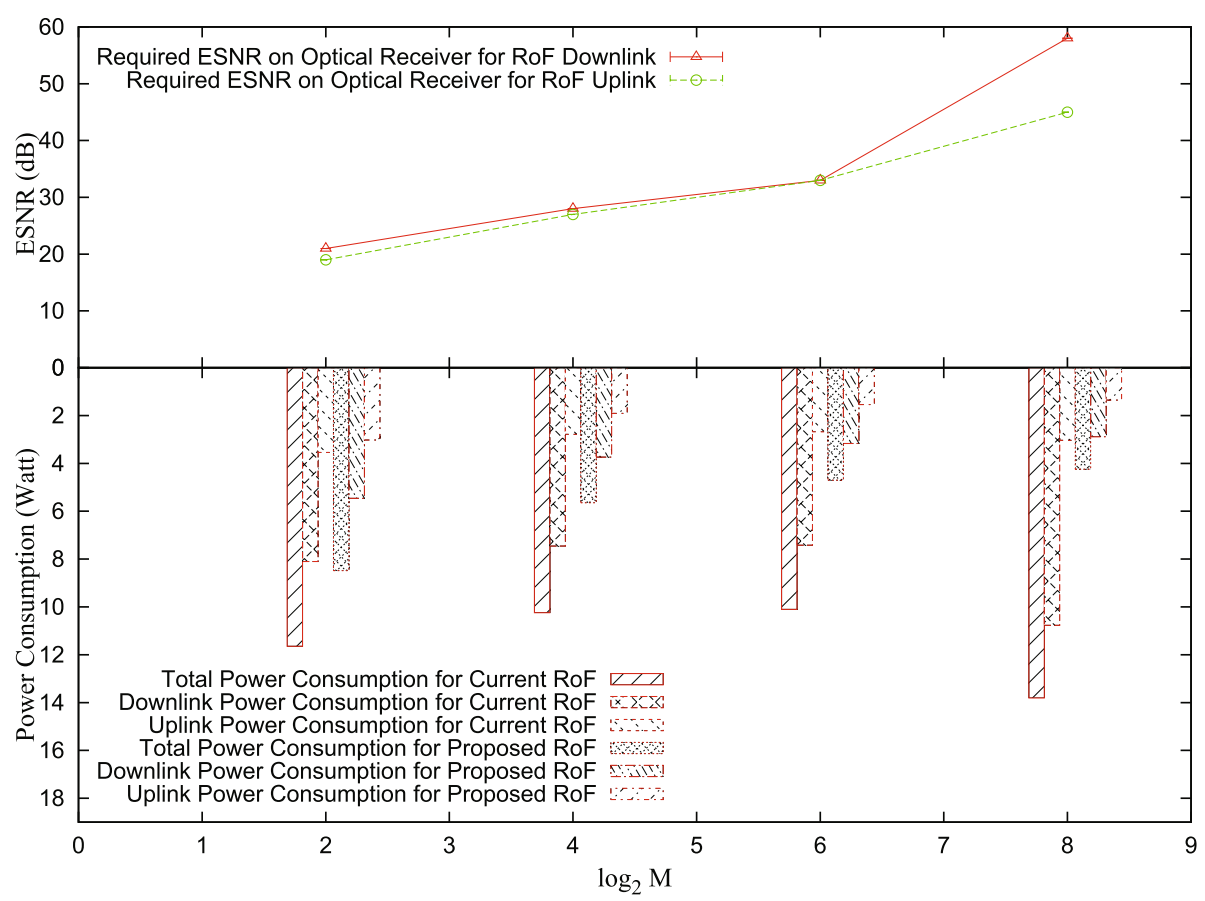

Fig. 4 Required ESNR on optical receiver and power consumption of RoF system for supporting a single BS with $V_{\pi}=6.5$ VS modulation level 
a value $6.626 \times 10^{-34}$; and $v$ is the optical frequency constant with a value $1.93 \times 10^{14} \mathrm{~Hz}$.

\section{Case study}

Case study is conducted to demonstrate the energy efficiency of the proposed MIMO-RoF system. Our goal is to observe the performance of the proposed RoF system in terms of power consumption. We are particularly interested in how the system can satisfy a given transmission rate with minimum transmit power by manipulating its modulation level scheme.

Without loss of generality, a single BS is connected. We set $D_{\text {down }}=30 \mathrm{Gbps}$ for downstream data transmission, and $D_{u p}=10 \mathrm{Gbps}$ for upstream data transmission. Each RAU/BS supports two sectors, each being equipped with 2 antennas of a $2 \times 2$ MIMO configuration. By taking the required ESNR in baseband as the simulation parameter in each scenario, we implemented V-BLAST for improving capacity gain and STBC for maximizing spatial diversity in the MIMO processing unit. Each radio channel includes 512 subcarriers, and candidate modulation levels include QPSK $(M=4), 16-$ QAM $(M=16)$, 64-QAM $(M=64)$, and 256-QAM $(M=256)$. For calculating OSNR, the distribution range is taken as $85 \mathrm{~km}$ and $100 \mathrm{~km}$ for the current and future C-RAN scenarios, respectively. The required voltage difference $V_{\pi}$ applied to the electrode of single-drive MZM is in the range of $6.5 \sim 10$ due to the upper bound of available OSNR. The BER requirement without using FEC at the receiver is
$10^{-6}$. The channel is assumed to be subject to AWGN and Rayleigh fading.

When $V_{\pi}$ of MZM is small as shown in Fig. 4, the 256-QAM modulation suffers significantly more signal distortion from RoF downlink than that from uplink, while the lower modulation levels of downlink suffer about the same distortion as that of uplink. This is due to the difference of the MIMO-OFDM module at the BS and the antenna sides, respectively, where a larger ESNR is required for achieving the given data rate. Similar observation is gained when $V_{\pi}$ of MZM is large as shown in Fig. 5, where the 64-QAM and 256-QAM modulations suffer more signal distortion from the RoF uplink than that from downlink, while the required ESNR of the lower modulations for downlink is only slightly affected.

For the current RoF system with small $V_{\pi}$ as in Fig. 4, using a higher level of modulation can generally improve the power efficiency, thanks to the improved coding efficiency. However, using 256-QAM breaks such a trend by consuming significantly more power since at this moment, the high SNR requirement at the receiver for the higher coding rate (256-QAM) cannot be satisfied due to the constant attenuation of the transmission line. Thus, it has to resort to increasing the transmit power for better SNR. Note that the increase of transmit power and level of modulation also introduces the performance impairment by PAPR, which explains the big jump of the power consumption. This is still true with large $V_{\pi}$ as in Fig. 5, where

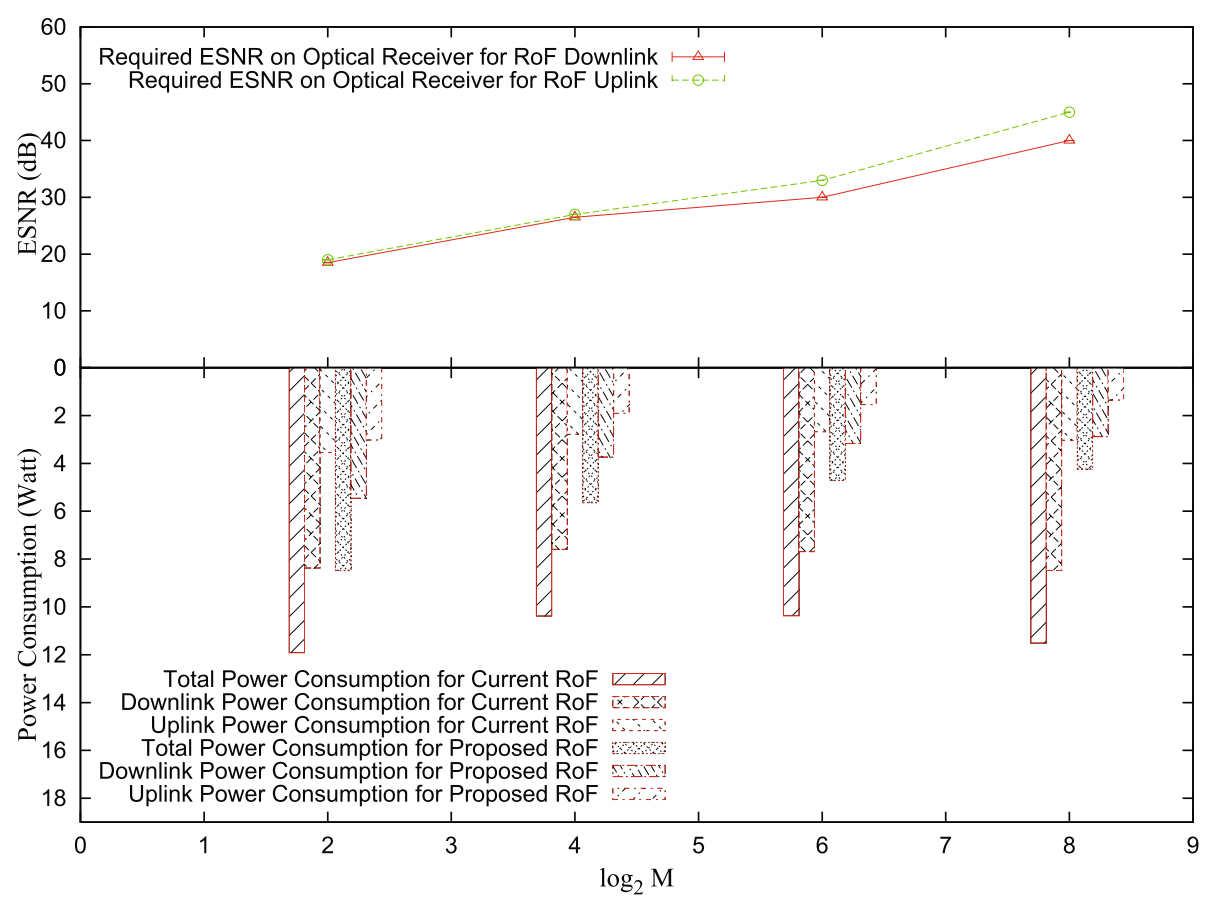

Fig. 5 Required ESNR on optical receiver and power consumption of RoF system for supporting a single BS with $V_{\pi}=10$ VS modulation level 
the highest power is consumed by QPSK, and the lowest power is also achieved by 16-QAM or 64-QAM.

For the proposed RoF system, on the other hand, it shows that the most aggressive modulation scheme 256QAM can be used for not only achieving the lowest power consumption in all cases for achieving the given data rate. This is due to the fact that the use of PDM and the PAPR reduction scheme can achieve better spectrum efficiency, such that the trend of higher-modulation-lower-power still holds when 256-QAM is being used. In summary, the proposed RoF system can save $27-69 \%$ power consumption compared with that of the current RoF system.

\section{Conclusions}

This paper introduced a novel adaptive radio-over-fiber (RoF) system for next-generation C-RAN in which energy consumption, capacity per wavelength, and distribution range are considered. By considering nonlinear distortion from both MZM and HPA, we first developed a $2 \times 2$ MIMO-OFDM baseband model for simulating the required ESNR of the RoF system. Then, we proposed a novel MIMO-RoF system for the next-generation C-RANs that can fit into the requirements of $5 \mathrm{G}$ mobile systems, where the OSNR analysis and its relation with ESNR was investigated. Lastly, a set of case studies was conducted aiming to observe the performance of the proposed RoF system in terms of its energy and bandwidth efficiency in presence of a given data rate. We demonstrated that the best energy efficiency and spectrum consumption can be achieved by using the most aggressive modulation format (256-QAM) with considerably less power consumption than the current RoF system.

\begin{abstract}
Abbreviations
ADC: Analog-to-digital convertor; AWG: Array waveguide grating; BBU: Baseband unit BER: Bit error rate; BS: Base station; C-RAN: Cloud radio access network; CD: Chromatic dispersion CW: Continuous wave; CO: Coherent optical CO-OFDM: Coherent optical OFDM; CoMP: Co-operative multi-point; CPRI: Common public radio interface; CU: Central unit; DAC: Digital-to-analog convertor; DAS: Distributed antenna systems; DC: Digital convertor; DFB: Distributed feedback; DSP: Digital signal processor; EDFA: Erbium-doped fiber amplifier; ESNR: Electrical signal-noise ratio; FEC: Forword error correction; HPA: High power amplifier; IP: Internet protocol; LR-PON: Long reach PON; LTE: Long-term evolution; MIMO: Orthogonal frequency division multiplexing; MIMO-OFDM: MIMO multiple-input and multiple-output; MZM: mach-zehnder modulator; OSNR: Optical signal-noise ratio; PAPR: Peak-to-average-ratio; PD: Photodiode; PDM: Polarization division multiplexing; PMD: Polarization mode dispersion; PON: Passive optical networks; QAM: Quadrature amplitude modulation; QoS: Quality of service; RoF: Radio-over-fiber; RRU: Remote radio unit; SCM: Subcarrier multiplexing; SMF: Single mode fiber; SSMF: Standard single mode fiber; STBC: Space-time block code; V-BLAST: Vertical bell labs layered space-time; WDM: Wavelength division multiplexing
\end{abstract}

\section{Acknowledgements}

This work was supported in part by the BK21 Plus Project (SW Human Resource Development Program for Supporting Smart Life) funded by the Ministry of Education, School of Computer Science and Engineering, Kyungpook National University, Korea, under Grant 21A20131600005, in part by the National Research Foundation of Korea grant funded by the Korean Government (2018R1D1A1B07051118), and in part by Kyungpook National University Research Fund, 2018.

\section{Authors' contributions}

All of the authors participated in the whole process of this research work and made considerable contributions, while with the following respective focus: (1) BW drawed all the figures and carried out the simulation/experiment part of this study. (2) LP mainly contributed in the proposing idea, verifying the illustrative figures for the proposed RoF transmission system, and revising the writing. (3) P-HH worked as an overall director in generating the major ideas, advising the system architecture, and organizing the overall paper writing. All authors read and approved the final manuscript.

\section{Competing interests}

The authors declare that they have no competing interests.

\section{Publisher's Note}

Springer Nature remains neutral with regard to jurisdictional claims in published maps and institutional affiliations.

\section{Author details}

${ }^{1}$ Department of Electrical and Computer Engineering University of Waterloo Canada, ON N2L3G1 Waterloo, Canada. ${ }^{2}$ School of Computer Science and Engineering, Kyungpook National University, 41566 Daegu, Republic of Korea.

Received: 23 April 2018 Accepted: 25 April 2019

Published online: 10 May 2019

References

1. F. Tian, et al., A survey on C-RAN security. IEEE Access. 55(1), 13372-13386 (2017)

2. D. Bojic, E. Sasaki, N. Cvijetic, W. Ting, J. Kuno, J. Lessmann, S. Schmid, H. Ishii, S. Nakamura, Advanced wireless and optical technologies for small-cell mobile backhaul with dynamic software defined management IEEE. Commun. Mag. 51(9), 86-93 (2013)

3. D. Chitimalla, et al., $5 \mathrm{G}$ fronthaul-latency and jitter studies of CPRI over ethernet. IEEE/OSA J. Opt. Commun. Netw. 9(2), 172-182 (2017)

4. D. Wake, et al., Radio over fiber link design for next generation wireless systems. J. Light. Technol. 28(16), 2456-2464 (2010)

5. H. Khodakarami, et al., Flexible optical networks: An energy efficiency perspective. J. Light. Technol. 32(21), 3356-3367 (2014)

6. D. Novak, Radio-over-fiber technologies for emerging wireless systems. IEEE J. Quantum Electron. 52(1), 1-11 (2016)

7. B. T. Teipen, et al., Adaptive data rates for flexible transceivers in optical networks. J. Netw. 7(5), 776-782 (2012)

8. J. E. Mitchell, Integrated wireless backhaul over optical access networks. J. Light. Technol. 32(20), 3373-3382 (2014)

9. I. S. Amiri, et al., two-by-two MIMO-OFDM-RoF generation and transmission of double $\mathrm{V}$-band signals using microring resonator system. Opt. Quant. Electron. 48(1) (2015)

10. M. Milosavljevic, et al., Demonstration of wireless backhauling over long-reach PONs. J. Light. Technol. 30(5), 811-817 (2012)

11. D. Lavery, et al., in Optical fiber communication conference and exposition (OFC/NFOEC). Bidirectional 10 Gbit/s long-reach WDM-PON using digital coherent receivers, (Los Angeles, 2011)

12. Litsyn, S. (Cambridge University Press, Cambridge, 2007)

13. K. P. Ho, Phase-modulated optical communication systems. (Springer Science + Business Media, Inc., 2005)

14. Y. London, D. Sadot, Nonlinear effects mitigation in coherent optical OFDM system in presence of high peak power. J. Light. Technol. 29(21), 3275-3281 (2011)

15. R. J. Essiambre, G. Kramer, P. J. Winzer, G. J. Foschini, B. Goebel, Capacity limits of optical fiber networks. J. Light. Technol. 28(4), 662-701 (2010)

16. B. S. Pillai, B. Sedighi, K. Guan, N. P. Anthapadmanabhan, W. Shieh, K. J. Hinton, R. S. Tucker, End-to-end energy modeling and analysis of long-haul coherent transmission systems. J. Light. Technol. 32(18), 3093-3111 (2014)

17. Gigabit-capable passive optical networks (GPON): Physical media dependent (PMD) Layer specification (2003). https://www.itu.int/rec/TREC-G.984.2-200303-I/en

18. R. Tucker, Green optical communications??Part I: Energy limitations in transport. J. Sel. Top. Quantum Electron. 17(2), 245-260 (2011) 